\title{
A High Resolution Melting Point Analysis Based Simplex PCR Assay Enables Reliable Identification of Clinically Relevant Candida Species
}

Gabor Fidler ${ }^{1}$, Eva Leiter², Sandor Kocsube ${ }^{3}$, Emese Tolnai ${ }^{1}$, Sandor Biro ${ }^{1}$, Melinda

Paholcsek ${ }^{1}$

1 University of Debrecen, Faculty of Medicine, Department of Human Genetics, Debrecen, Hungary

2University of Debrecen, Faculty of Science and Technology, Department of Biotechnology and Mycrobiology, Debrecen, Hungary

3University of Szeged, Faculty of Science and Informatics, Department of Microbiology, Szeged, Hungary

\section{INTRODUCTION}

The genus Candida corresponds to the most important opportunistic mycoses in the world. Nearly $95 \%$ of invasive candidemia is caused by Candida albicans, C. glabrata, C. parapsilosis, $C$. tropicalis and Candida krusei. The remaining 5\% of candidemias are caused by Candida guilliermondii, $C$. dubliniensis provoking superinfections due to their low sensitivity to broad-spectrum antifungals. The correct identification of Candida species is of high importance because knowledge of species may influence adequate antifungal therapy. Here we introduce a PCR method which can differentiate among seven clinically relevant Candida species: Candida albicans, Candida glabrata, Candida parapsilosis, Candida tropicalis, Candida krusei, Candida guilliermondii, Candida dubliniensis using a single primer pair for the amplification followed by a post-PCR Tm calling assay coupled with high-resolution melting analysis. The denaturation of the target DNA amplicons defines the characteristic melting peaks and the shape of the derivative melting curves which represent the unique taxonomic footprints of the 7 Candida species.

\section{METHODS}

Primer design: We targeted the Candida beta-tubulin genes, and screened the sequences for melting domains covering enough mismatches to proper discrimination.

Real-time PCR platforms: Real-time PCR amplification were optimized for three different Roche PCR platforms: (P1) LightCycler® 96, (P2) LightCycler® Nano and (P3) LightCycler® 2.0 Instrument. For the amplification the Roche LightCycler 480 High Resolution Melting Master was used.

Discriminatory power, specificity and limit of detection: 7 Candida reference strains and 38 pre-characterized Candida clinical strains were investigated using our method. Crossreactivity was tested on 34 Aspergillus, 4 Fusarium, 10 Grampositive and 6 Gram-negative bacteria strains and on human genomic DNA. Limit of detection was measured using whole blood samples collected from healthy volunteers spiked with Candida cells in a 6 log range.

\section{RESULTS}

We were able to create 7 unique melting clusters, representing the different Candida species. All of the 38 Candida clinical samples showed melting peaks in a close range of the reference strains. No cross-amplification was detected with human genomic DNA nor with bacteria and Fusarium strains. Contrarily some Aspergillus strains showed mild cross reaction with melting peaks above the Candida melting clusters. The analytical sensitivity, the lowest concentration of template DNA where conclusive melting peaks and HRM curves were attainable, proved to be 10 cells $/ 1 \mathrm{ml}$ whole blood on all platforms.

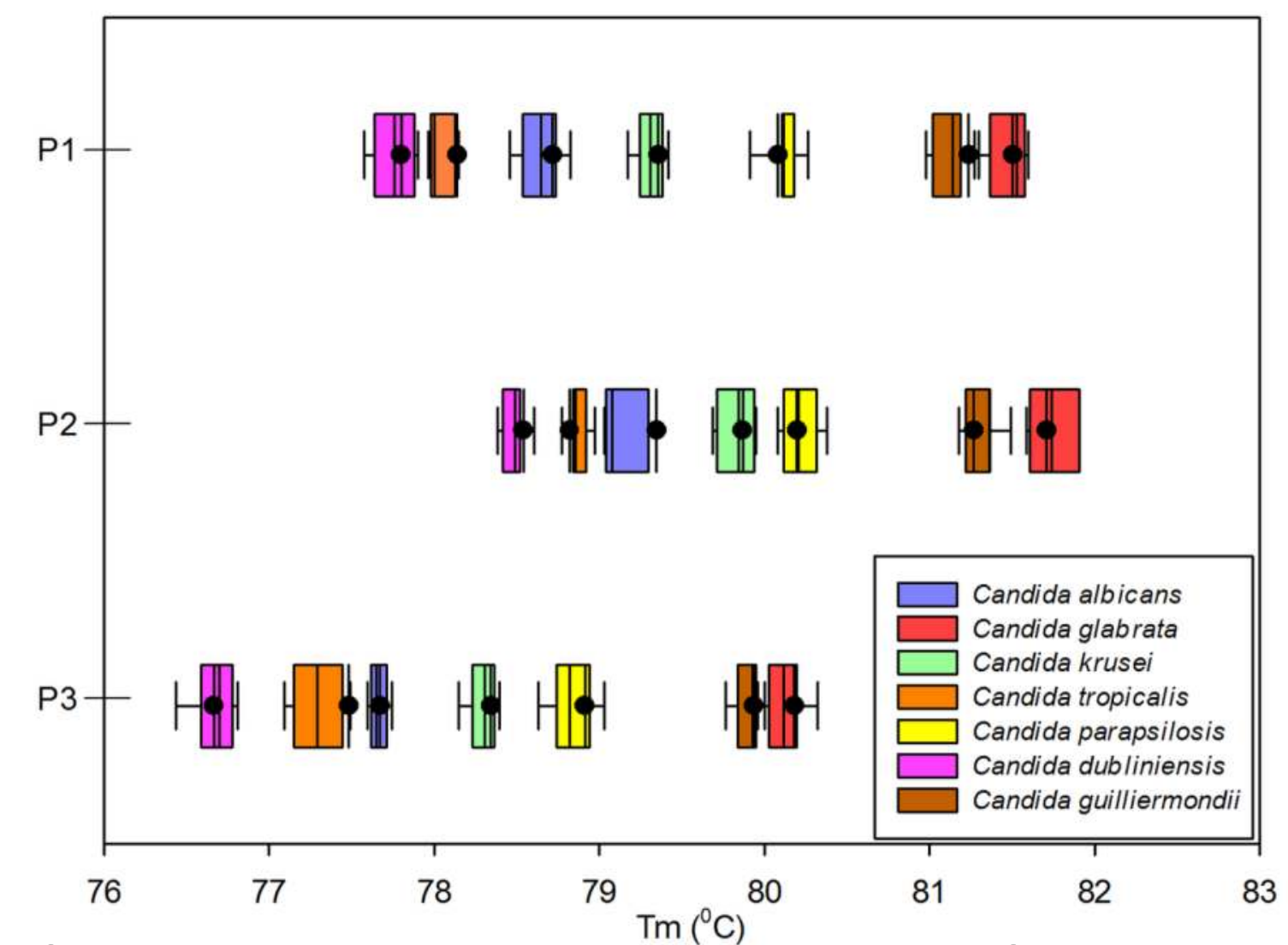

Candida melting clusters on different PCR platforms: Roche LightCycler 96 (P1), Roche LightCycler Nano (P2), Roche LightCycler 2.0 (P3)

\section{CONCLUSION}

Here we describe our CanTub-simplex HRM PCR method which can identify 7 clinically relevant Candida species. It is sensitive, cost-effective and can provide results with a short turnaround time. The clinical validation, which includes testing our assay on hemocultures, biofilms and blood samples of patients with fevered neutropenia is now in progress.

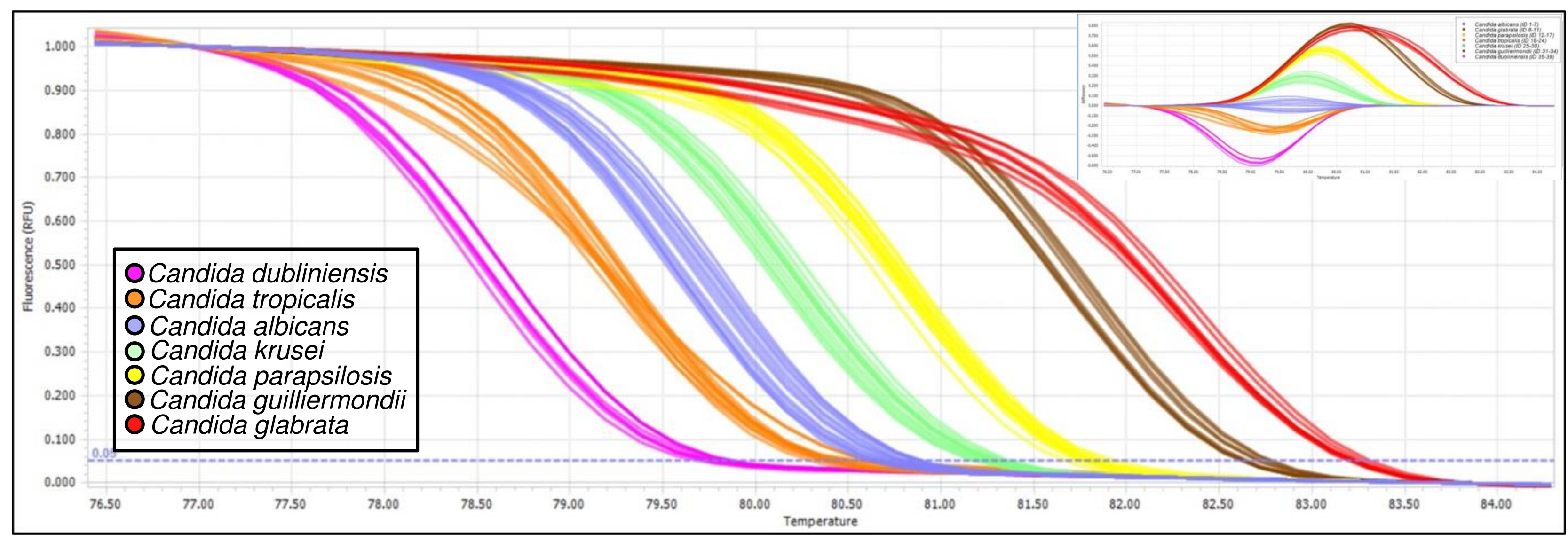

